\title{
Estandarización del método espectroscópico UV-Visible para la determinación del contenido de asfaltenos en crudos pesados
}

\author{
Standardization of the spectroscopy method UV-Visible to \\ determine the asphaltene content in heavy crude oils
}

\author{
Cesar Augusto Bernal Samaniego \\ Universidad de Panamá, Nano Dispersions Tecnology,Inc \\ cesarbernal10@gmail.com \\ Alexandra Miguelina Castro Rojas \\ Nano Dispersions Tecnology, Inc. \\ acastro@nanodt.com \\ Matritza Ramos de Leone \\ Universidad de Panamá \\ mrleone2@yahoo.com \\ Antonio Luis Cárdenas Rodulfo \\ Nano Dispersions Tecnology, Inc. \\ acardenas@nanodt.com
}

\section{Resumen}

Este estudio pretende implementar una metodología para lograr determinar el contenido de asfaltenos en crudos pesados resultantes de la aplicación del proceso de desasfaltación para disminuir el tiempo de medición y reducir el volumen del disolvente en comparación con el método convencional ASTM D 3279-07. Se logró mejorar el tiempo de medición se redujo a menos de 3 horas en vez de 2 días, mientras que el volumen del disolvente se disminuyó a una cuarta parte.

\section{Palabras clave}

Cuantificación, hidrocarburos, petróleo, normas ASTM, desasfaltado.

\begin{abstract}
This study intends to implement a methodology to determine the asphaltene content in heavy crude oils that have been subjected to a deasphalting process, in order to reduce the measurement time and the solvent volume in comparison to the conventional method ASTM D 3279-07 (Standard Test Method for $n$-heptane insoluble). As a result, the measurement time was reduced to less than 3 hours instead of 2 days, and the solvent volume was reduced to $25 \%$ of the usual requirement.
\end{abstract}




\section{Keywords}

Quantification, hydrocarbon, petroleum, ASTM rules, deasphalting. 


\section{INTRODUCCIÓN}

En la actualidad, el mundo continúa su dependencia energética de los hidrocarburos derivados del petróleo, por lo que la investigación en el área petrolera se ha mantenido y ha evolucionado para dar solución a los diferentes problemas que se han presentado en relación con la producción, el procesamiento y el transporte de crudos.

Dentro de la composición del crudo, existe un grupo de moléculas complejas que han sido denominadas como asfaltenos; estos son los compuestos más pesados. La precipitación y deposición de asfaltenos puede ocurrir durante la producción y procesamiento de crudo, cosa que representa un problema serio y costoso (1). Los depósitos asfalténicos se pueden formar en el reservorio, en la tubería del pozo productor e incluso pueden ser arrastrados a través de las líneas de flujo hasta los separadores; además, causan taponamiento en tuberías, platos de torres y otros equipos; son responsables del envenenamiento de catalizadores debido al alto contenido de metales que poseen y también contribuyen a incrementar la viscosidad de los crudos (2).

Por otra parte, si se diluye el crudo o algún residuo pesado con disolventes o fracciones petroleras para disminuir su viscosidad y facilitar el transporte, cambia la composición del medio y en consecuencia la solubilidad de los agregados asfalténicos, estimulando su deposición. Disolventes poco aromáticos o muy parafínicos disminuyen la peptización de los asfaltenos en el crudo o fracción. Como puede notarse, la composición de crudo o derivado es determinante a la hora de seleccionar el diluente adecuado $(3,4)$

El método tradicional empleado para la cuantificación del porcentaje de asfalteno es el método gravimétrico, en el cual los asfaltenos se precipitan y lavan con disolventes apropiados, y el precipitado se pesa al final. Hay una gran cantidad de disolventes que precipitan los asfaltenos, siendo los más usados para su determinación el pentano o el heptano (5). Este es un método largo y engorroso (puede durar de dos a tres días, dependiendo del crudo), además consume mucho reactivo y por ello se busca implementar un método que permita determinar la concentración de asfaltenos de manera más rápida, sencilla y con menor consumo de reactivos. Una vía es usando mediciones de espectroscopia UV-Visible (6). En este caso, la posibilidad de usar UV-Visible se presenta como un potencial método para la cuantificación del contenido de asfaltenos de una manera más rápida y menos complicada experimentalmente que los métodos tradicionales empleados. En la literatura existen artículos que describen la determinación del contenido de asfaltenos por el método UV-Visible.

Estos, por lo general, se han usado para medir diferencias entre cambios en la concentración de asfaltenos en una misma disolución, por ejemplo, en estudios de adsorción de asfaltenos (7). Otros investigadores han propuesto un método que permite cuantificar el porcentaje de asfaltenos considerando una gran cantidad de crudos, basándose en medir la diferencia entre el crudo inicial diluido y los maltenos luego de precipitar los asfaltenos, a dos longitudes de onda distintas para corregir por línea de base y diferencias en los índices de refracción $(8,9)$. La existencia de grupos aromáticos en la estructura de los asfaltenos, que son una familia más que una molécula particular, ayuda a su determinación por UV-Visible. 
En este trabajo se pretende realizar la estandarización de un crudo pesado en específico, mientras que los trabajos anteriores empleaban un grupo de crudos. La idea de hacerlo más específico es que se obtenga una mayor exactitud y precisión, además de resolver una problemática del quehacer laboral, pues era uno de los crudos más trabajados en la empresa.

\section{MATERIALES Y MÉTODOS}

\subsection{Materiales}

Los asfaltenos fueron obtenidos a partir del crudo colombiano, proporcionado por la empresa Pacific Rubiales Energy y denominado como crudo pesado Quifa, el cual posee un 10,2\% de asfaltenos. Es por ello que cuando se hable de crudo es en general, mientras que el crudo Quifa es con el que se trabajó específicamente. Los disolventes utilizados son marca Merck con un 99\% pureza. Para realizar las mediciones se empleó un espectrofotómetro UV-Visible modelo Cary 60 de Agilent Technologies con un rango de longitud de onda de 190 - $1100 \mathrm{~nm}$.

\subsection{Metodología}

La metodología empleada en la realización de este trabajo se desarrolló de la siguiente manera.

\subsubsection{Obtención de muestras de crudo desasfaltado y asfaltenos}

Como primer paso, para la generación de muestras de análisis se empleó el proceso de desasfaltado de crudo patentado por la empresa NanoDispersions Technology (Process of reducing viscosity of heavy crude oil by removal of asphaltene using a precipitating agent), denominado posteriormente CAR (Colloidal Asphaltene Removal), bajo la solicitud de patente número US20130264247 (10). Las muestras resultantes obtenidas en este proceso corresponden al DAO o crudo desasfaltado; esto implica la remoción de los asfaltenos más resinas que contiene el crudo.

\subsubsection{Preparación de muestras para obtener la curva de calibración}

El crudo Quifa y el correspondiente desasfaltado fueron enviados a un laboratorio externo para su análisis, con el fin de determinar la cantidad base de asfaltenos presente. Se obtuvo que en el DAO de crudo Quifa se encontraba 0,2\% de asfalteno, mientras que para el crudo Quifa inicial se obtuvo un $10,2 \%$ de asfaltenos.

Las mezclas se prepararon de acuerdo con el siguiente procedimiento:

1. Se pesaron en tubos de centrífuga las cantidades correspondientes de crudo desasfaltado y de crudo original (sin desasfaltar).

2. Las muestras se colocaron en el ultrasonido por un periodo de 60 minutos hasta lograr su homogenización, para obtener una las mezclas de acuerdo con las composiciones que se indican en la Tabla 1 para el crudo Quifa. 
3. Una vez obtenidas todas las mezclas, se procedió a preparar las respectivas soluciones a la concentración de 0,4 \% (4 000 ppm) utilizando cloroformo como disolvente.

4. Se realizaron barridos de longitud de onda para evaluar las diferencias en las curvas de absorción de las mezclas.

5. Se realizaron las mediciones de absorbancia en función de la concentración del contenido de asfaltenos para la elaboración posterior de las curvas de calibración.

6. Se realizaron las curvas de calibración a las longitudes de onda de 400, 500, 600, 700 y $800 \mathrm{~nm}$. Posteriormente, se procede a realizar el método de Kharrat et al., en donde se realiza una diferencia entre las absorbancias obtenidas a dos diferentes longitudes de onda, con la finalidad de mejorar la linealidad y aumentar el coeficiente de correlación (8).

Tabla 1. Mezclas de crudo desasfaltado (DAO) y crudo Quifa.

\begin{tabular}{ccc}
\hline \multicolumn{3}{c}{ Crudo Quifa } \\
\hline DAO $(\mathrm{g})$ & Crudo $(\mathrm{g})$ & $\begin{array}{c}\text { Porcentaje de Asfalte- } \\
\text { nos }(\%)\end{array}$ \\
15,02 & 0 & 0,2 \\
13,5 & 1,5 & 1,2 \\
12,05 & 3,01 & 2,2 \\
10,49 & 4,50 & 3,2 \\
9,02 & 6,00 & 4,2 \\
7,49 & 7,55 & 5,2 \\
6,03 & 9,00 & 6,2 \\
4,51 & 10,51 & 7,2 \\
3,01 & 12,00 & 8,2 \\
1,51 & 13,56 & 9,5 \\
0 & 15,20 & 10,2 \\
\hline
\end{tabular}

\subsubsection{Correlación entre la concentración de las muestras y la espectroscopia} óptica. Ley de Beer

Inicialmente, se procedió a preparar disoluciones a diferentes concentraciones de asfaltenos Quifa para realizar las curvas de calibración y verificar la validez, en cada caso, del uso del método espectroscópico; es decir, el cumplimiento de la ley de Beer. Se preparó una disolución madre de 400 ppm $(0,04 \%$ p/v). De la disolución madre se tomaron las alícuotas respectivas para generar las disoluciones de concentraciones 80 ppm, 60 ppm, 40 ppm, 20 ppm y 8 ppm. Para cada solución, se realizaron las respectivas medidas de absorbancia y se realizaron barridos de longitudes de onda en el rango de $200 \mathrm{~nm}$ a $800 \mathrm{~nm}$. Además de las disoluciones de asfaltenos, se prepararon disoluciones de crudo Quifa y crudo desasfaltado para realizar barridos en el rango de $200 \mathrm{~nm}$ a $800 \mathrm{~nm}$. 


\subsubsection{Cuantificación de los porcentajes de asfaltenos en las muestras problema}

Las muestras de crudo desasfaltado (DAO) (relaciones 1:1, 1:3), fueron analizadas en el UV-Visible de acuerdo con el siguiente procedimiento: se prepararon disoluciones de concentraciones de $0,04 \%$ (400 ppm) y 0,4\% (4000 ppm) y se midieron a longitudes de 600 y $700 \mathrm{~nm}$. Cada absorbancia fue correlacionada con los respectivos datos en la curva de calibración para obtener el correspondiente porcentaje de asfalteno. Cada muestra fue sometida al análisis por medio de la norma ASTM D 3279-07 para establecer comparaciones entre el método espectroscópico y el método estándar.

\section{RESULTADOS Y DISCUSIÓN}

\subsection{Obtención de muestras de crudo desasfaltado y asfaltenos}

Como se ha visto, los métodos de cuantificación del porcentaje de asfaltenos se basan en la utilización de un exceso de n-parafinas para una precipitación total. Sin embargo, se ha demostrado que dependiendo de la composición del crudo (distribución de sus componentes en función de los asfaltenos), se pueden utilizar cantidades moderadas de disolvente para alcanzar la precipitación total de los asfaltenos y, por ende, la obtención de un crudo desasfaltado con mejores propiedades en cuanto a la reducción de viscosidad y menores cantidades de azufre entre otras.

Se aplicó en este trabajo el proceso CAR a escala de laboratorio con el propósito de generar muestras problema de crudo desasfaltado a partir del crudo Quifa original, con el fin de determinar el contenido de asfaltenos mediante el método UV-visible propuesto y mediante la norma ASTM correspondiente para, con esto, establecer comparaciones entre ambos casos.

Las relaciones crudo:disolvente, utilizadas de acuerdo con el proceso CAR, fueron de 1:1 y 1:3, lo cual permitió la obtención de cantidades de asfaltenos comparables a las obtenidas mediante el método de precipitación convencional con relaciones 1:40 crudo:disolvente.

Empleando el proceso CAR usando una relación 1:1, se obtiene una distribución de $14,5 \%$ asfaltenos y $85,5 \%$ de crudo desasfaltado. En el proceso, los asfaltenos separados se encuentran interaccionando con una cantidad significativa de resinas que coprecipitan. Al someter a los asfaltenos a un proceso de lavado o purificación en un sistema Soxhlet, se obtuvo que las resinas coprecipitadas corresponden aproximadamente a un $4 \%$ del total de asfaltenos impuros. Esto permite obtener un crudo desasfaltado con una remoción de asfaltenos cerca del 100\%. Las resinas coprecipitadas con los asfaltenos no fueron mezcladas con el crudo desasfaltado ya que, en el proceso real, este aspecto no se considera y el mismo no interfiere con los resultados, ya que en ningún caso se utilizan los asfaltenos (con o sin resinas) para las determinaciones cuantitativas.

El crudo desasfaltado es la muestra que es sometida a análisis usando el método espectroscópico y el método gravimétrico (norma ASTM) para estimar el contenido de asfaltenos remanentes en dicha muestra. 


\subsection{Aplicación del método UV-visible}

Para evaluar el método espectroscópico en la determinación de la cantidad de asfahltenos presentes en crudos o crudos desasfaltados, se requirió de muestras con diferentes porcentajes de asfaltenos, por lo que se realizaron mezclas entre el crudo desasfaltado y el crudo Quifa, en diferentes proporciones, donde la cantidad máxima de asfaltenos estuvo dada por la cantidad de asfaltenos que posee el crudo Quifa. Dichas mezclas fueron denominadas crudos sintéticos.

Se realizaron barridos en el rango de longitudes de onda de 200-800 $\mathrm{nm}$ a las soluciones de crudos sintéticos, preparadas a la concentración de 20 ppm de crudo sintético (Fig. 2).

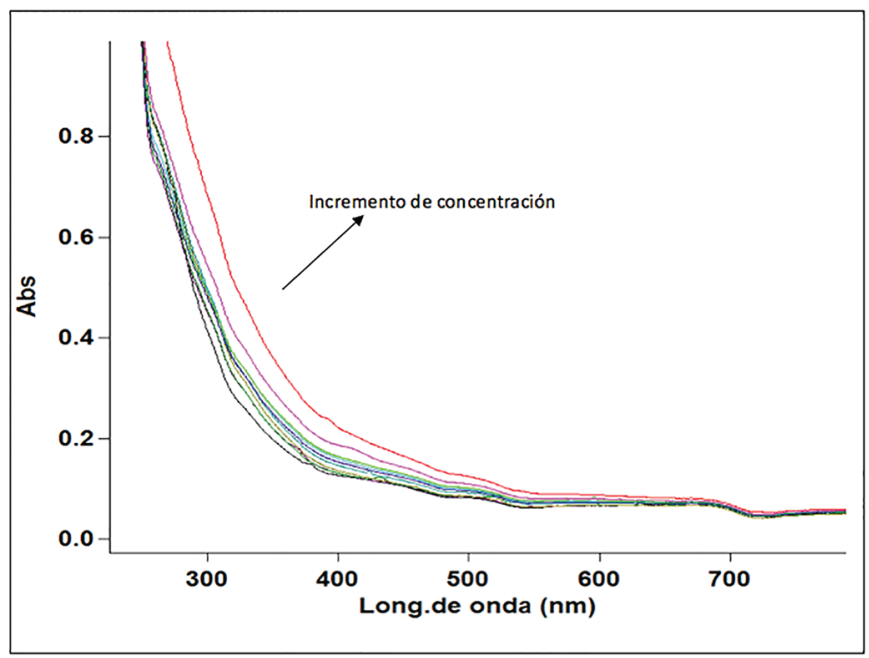

Figura 1. Curvas de barrido de longitud de ondas en el rango de $200 \mathrm{~nm}$ a $800 \mathrm{~nm}$ para mezclas de crudo Quifa.

En la Fig. 1 se muestra una gráfica comparativa, en la cual se pueden notar las diferencias entre las curvas, las cuales se deben a la variación en el contenido de asfaltenos presente en cada caso. A medida que aumenta el contenido de asfaltenos (de $0,2 \%$ a $10,2 \%$ ), hay un aumento de la absorbancia para cada longitud de onda.

A partir de las muestras de crudo sintético preparadas, se construyeron curvas de calibración a diferentes longitudes de onda, para la cuantificación del porcentaje de asfaltenos en las muestras problema.

Debido a las bajas absorbancias registradas entre 400 y $700 \mathrm{~nm}$ (Fig. 2) para 20 ppm de crudo, se trabajó con concentraciones de las mezclas de 400 ppm (Fig. 3) y 4000 ppm (Fig. 5).

De las gráficas de la Fig. 2 se puede observar que los datos no se ajustan de manera perfecta en ninguna de las longitudes de onda consideradas, lo cual se refleja en el coeficiente de correlación. 


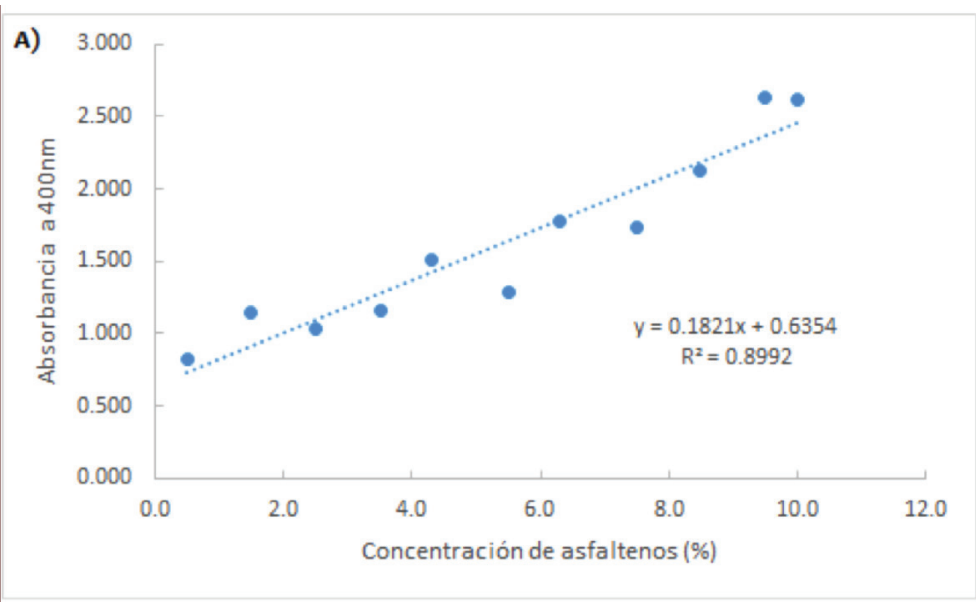

\section{B)}

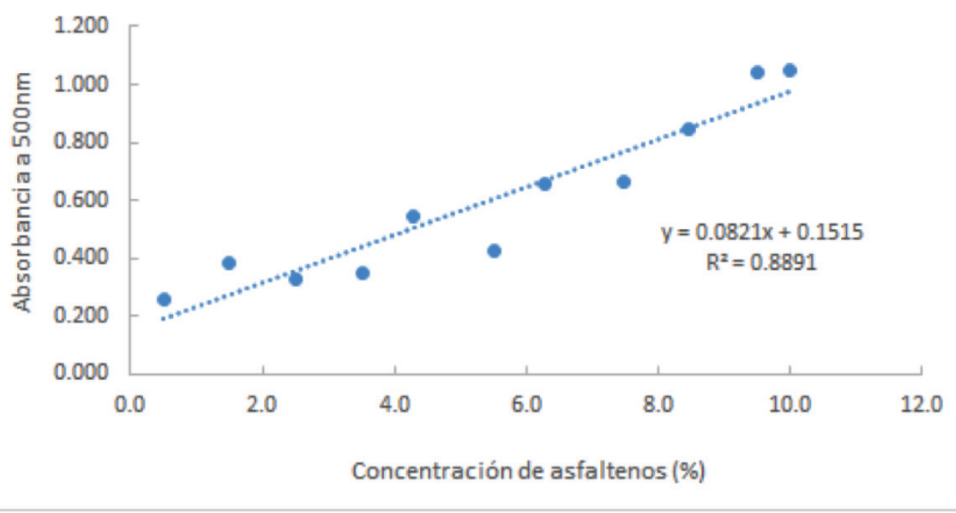

c)

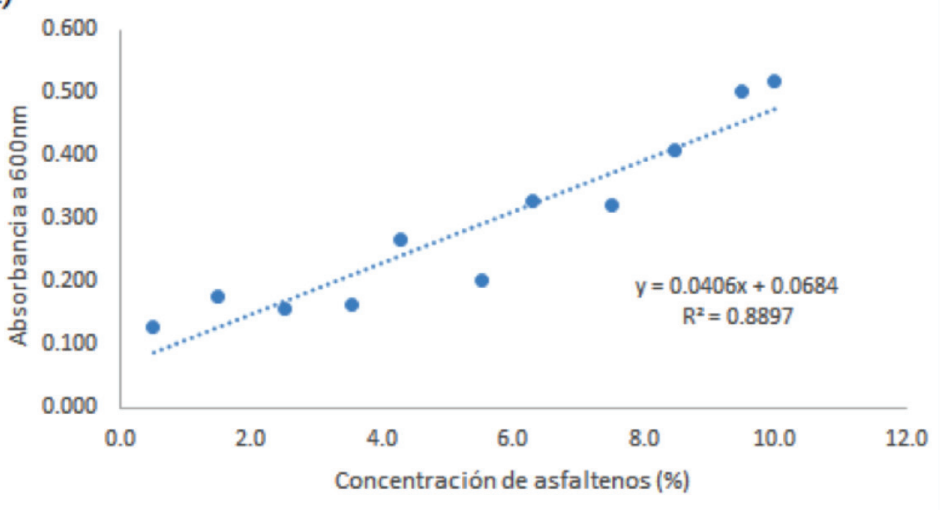

Figura 2. Absorbancias de los crudos sintéticos a $400 \mathrm{ppm}$. A) Medidas a 400nm B) Medida a $500 \mathrm{~nm}$ C) Medida a $600 \mathrm{~nm}$. 
Para este tipo de correlaciones, en las cuales se establece relación entre la absorbancia de una muestra global en función de la composición de uno de sus componentes, algunos investigadores han desarrollado metodologías para disminuir las dispersiones en los datos (11): consideraron la diferencia de los datos a dos longitudes de onda para cada concentración. Aplicando este método se obtuvieron las curvas que se muestran en la Fig. 3.
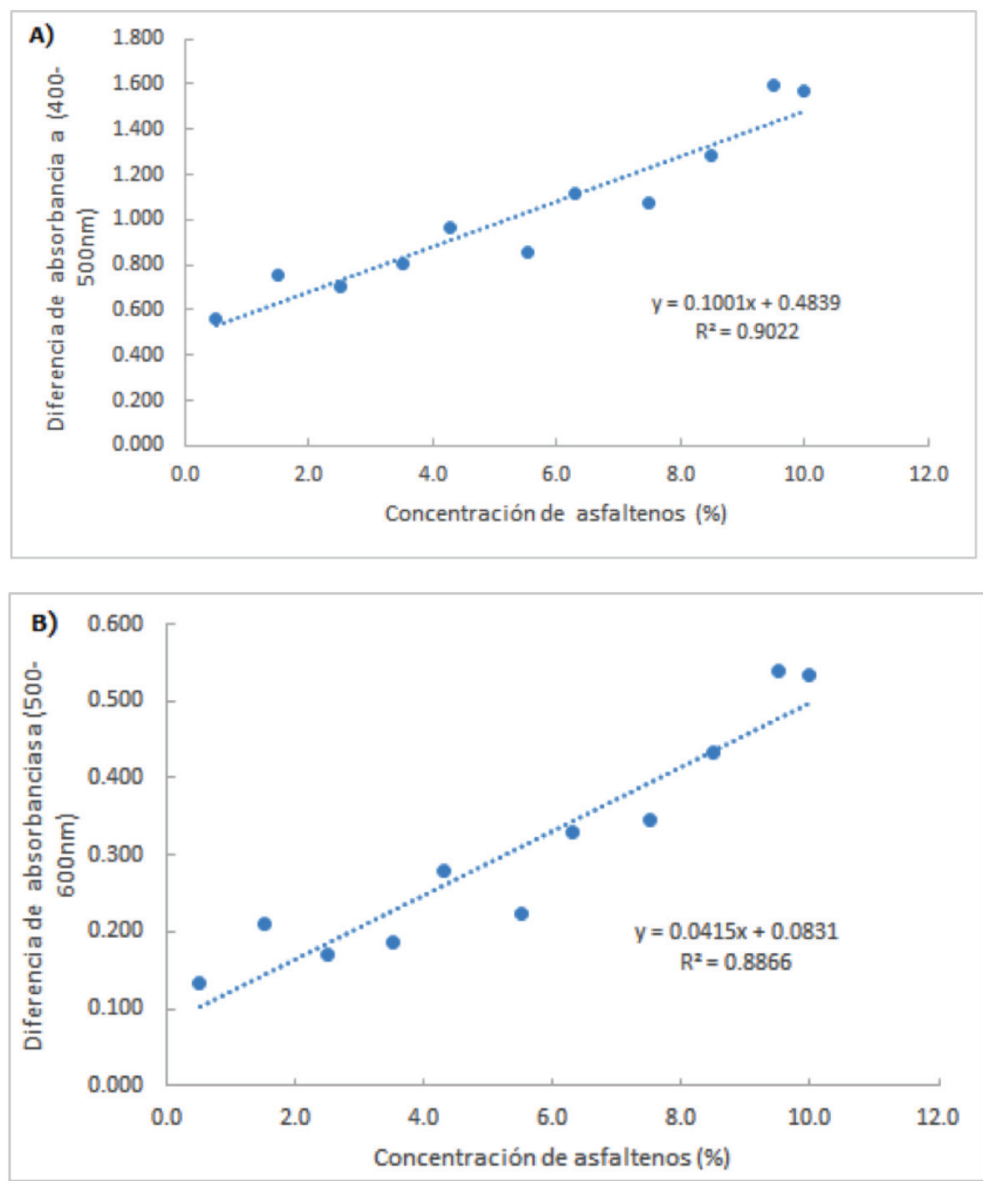

Figura 3. Diferencia de absorbancias de los crudos sintéticos Quifa a 400 ppm. A) Diferencias mediadas entre 400-500nm B) Diferencias mediadas entre 500-600nm.

De las curvas de la Fig. 3, con la aplicación de la diferencia de las absorbancias a las correspondientes longitudes onda, se puede notar que disminuyó la dispersión de los datos y el coeficiente de determinación incrementó, obteniéndose el mejor resultado para la diferencia entre 500 y $600 \mathrm{~nm}$.

Con el propósito de mejorar las curvas obtenidas, se preparó otra serie de soluciones a 4000 ppm de concentraciones y se evaluó el comportamiento de las curvas a las longitudes de onda de 500, 600 y $700 \mathrm{~nm}$ (Fig. 4). 

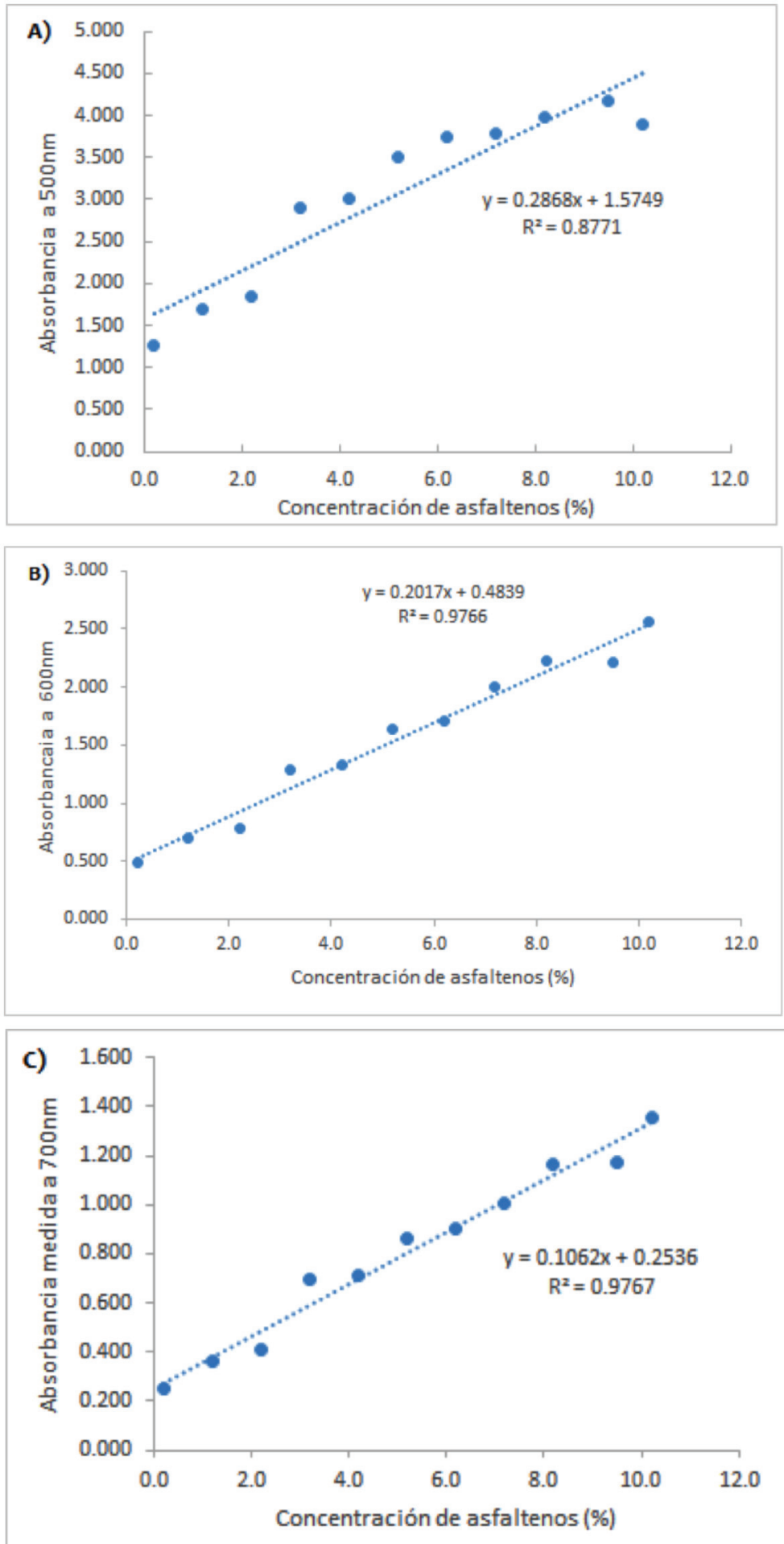

Figura 4. Absorbancias de los crudos sintéticos Quifa a 4000 ppm. A) Medidas a 500nm B) Medidas a $600 \mathrm{~nm}$ y C) Medidas a $700 \mathrm{~nm}$. 
En la Fig. 4, se puede notar que en la concentración de 4000 ppm, la dispersión de los datos disminuyó con respecto a las curvas realizadas a $400 \mathrm{ppm}$. Al igual que en el caso anterior, se aplicó la diferencia de las absorbancias y las curvas obtenidas se muestran en la Fig. 5. Para la diferencia de las absorbancias entre las longitudes de onda de 500 y $600 \mathrm{~nm}$ la dispersión de los datos incrementó, mientras que para la diferencia entre 600 y $700 \mathrm{~nm}$ la dispersión es similar que para los casos por separado.
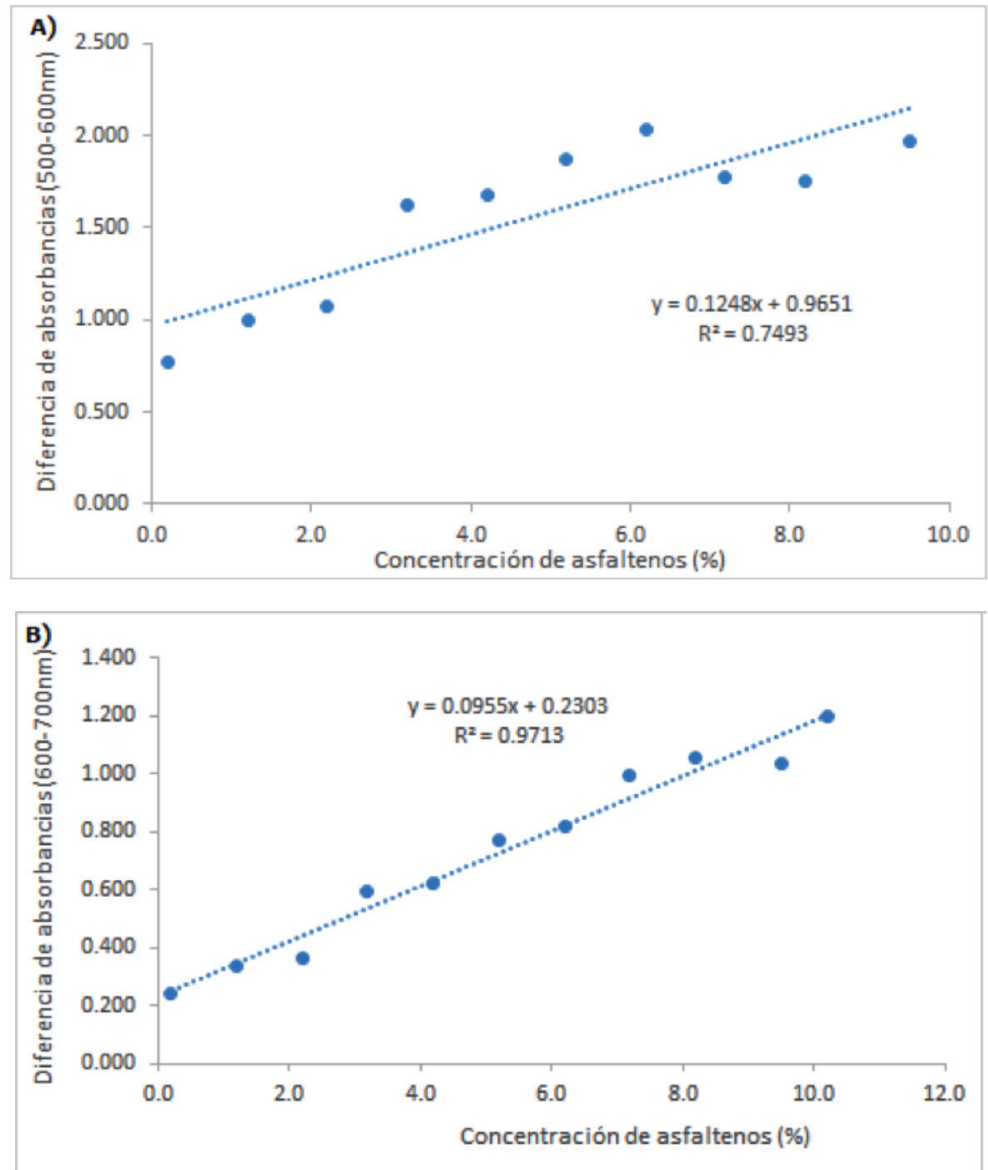

Figura 5. Diferencia de las curvas de absorbancia en función de la concentración de asfaltenos en la mezcla de Crudo Quifa a 4000 ppm.

El hecho de utilizar para un mismo crudo la medida de la concentración a una longitud de onda $700 \mathrm{~nm}$ y a una diferencia de longitudes de onda (600-700 nm) no tiene una influencia significativa sobre la calidad de la medición, tal como lo indican los coeficientes de correlación (R2). Esta diferencia se ha observado cuando se aplica una técnica similar con diferentes tipos de crudo con la intención de medir con una curva la concentración de asfaltenos de crudos distintos (8): es posible tener una buena curva de medición de asfaltenos para el crudo Quifa con la medida a una sola longitud de onda. 


\subsection{Comparación entre UV-visible y método ASTM D3279.}

A continuación, se realiza la comparación entre los resultados obtenidos mediante el método UV-visible y método ASTM. En la Tabla 2 se indican los resultados obtenidos para cada relación crudo:disolvente utilizada.

Tabla 2. Comparación entre los resultados obtenidos mediante el método UV- visible medido por la diferencia de absorbancias a las longitudes $\lambda=500-600 \mathrm{~nm}$ a las concentraciones de 400 ppm y 4000 ppm y el método ASTM D3279 para la relación 1:1 y 1:3 promedio.

\begin{tabular}{cccc}
\hline Pruebas Promedio & $\begin{array}{c}\text { Porcentaje de asfaltenos } \\
\text { UV-visible 400 ppm (\%) }\end{array}$ & $\begin{array}{c}\text { Porcentaje de asfaltenos } \\
\text { UV-visible 4000 ppm (\%) }\end{array}$ & $\begin{array}{c}\text { Porcentaje de asfaltenos } \\
\text { ASTM 3279 }(\%)\end{array}$ \\
\hline Relación 1:1 & $2,4 \pm 0,8$ & $2,3 \pm 0,2$ & $3,4 \pm 0,5$ \\
Relación 1:3 & $0,4 \pm 0,2$ & $0,5 \pm$ NA & $1,6 \pm 0,5$ \\
\hline
\end{tabular}

Hay una diferencia entre los asfaltenos obtenidos mediante la norma ASTM D 3279-09 y el método espectroscópico. Esa diferencia puede deberse a que con el método gravimétrico (ASTM D 3279-09) se precipitan algunas resinas con los asfaltenos, lo que puede incidir en una medida de asfaltenos superior a los medidos con el método espectroscópico, que solo toma en cuenta las moléculas con núcleos aromáticos. Esto sugiere que los métodos comparados no necesariamente miden exactamente lo mismo. Sin embargo, las tendencias observadas son similares; es decir, que en ambos métodos se observa un descenso de la cantidad de asfaltenos cuando se utiliza una relación de tratamiento 1:3 de crudo a solvente comparada con la relación 1:1 de crudo a solvente. Esta tendencia es la esperada, ya que cuando hay mayor cantidad de solvente, hay una mayor precipitación de asfaltenos.

Estos resultados indican que se puede usar el método espectrofotométrico para controlar el comportamiento de una unidad de desasfaltado, pero siempre considerando la brecha que existe con el método gravimétrico ASTM D 3279-09, que se puede utilizar como referencia en caso de ser exigido por algún cliente o por la venta de productos. Este método ASTM D 3279-09 no es el único método para determinar asfaltenos mediante precipitación; se pueden también usar otros, como por ejemplo el ASTM D 6560-00 o el IP 143 como referencia (12). También es posible desarrollar una correlación entre el método espectrofotométrico y el método gravimétrico.

\section{CONCLUSIONES}

El estudio realizado permite obtener las conclusiones siguientes:

- Es posible medir la cantidad de asfaltenos del crudo Quifa utilizando un método espectroscópico con buena precisión. Las curvas obtenidas en longitudes de onda de 700 y $800 \mathrm{~nm}$ permiten obtener un coeficiente de correlación superior al 0,95. 
- El uso del método que considera la diferencia de longitudes de onda también permite obtener buenos resultados, similares a los que se obtienen con la medición de una longitud de onda.

- Es posible utilizar el método espectroscópico para controlar a una planta de desasfaltado debido a que es posible seguir la tendencia de la precipitación de asfaltenos al cambiar las variables del proceso como la relación crudo/disolvente.

- Hay una diferencia entre los resultados obtenidos por el método gravimétrico (ASTM D 3279-07) y el espectroscópico; sin embargo, existe la posibilidad de hacer una correlación que permita relacionar los resultados de uno y otro método.

\section{REFERENCIAS}

1. Yen T, Chilingarian G. Development in Petroleum Science Asphaltenes and asphaltes. First Edit. USA: Elsevier . 2000. Chapter 2: The realms and definitions of asphaltenes. p. 7-645.

2. Delgado J . Cuadernos FIRP. 2da ed. Mérida,Venezuela: Universidad de Andes. Facultad de ingeniería. 2015. Precipitación de asfaltenos. p. 1-29.

3. Delgado J. Cuadernos FIRP. 1 era. Mérida, Venezuela: Universidad de los Andes, Facultad de ingenieria. 2006. Asfaltenos composición, agregación, precipitación. laboratorio de formulación, interfases, reología y procesos p. 38.

4. Goual L. Crude Oil Emulsions- Composition Stability and Characterization.. USA: University of Wyoming. 2012. Petroleum Asphaltenes. p. 27-40.

5. "Standard Test Method for n-Hepptane Insolubles", ASTM D3279-07, an American National Standard, vol. 64. 2008. p. 3-5.

6. Delgado J. Cuadernos FIRP. Mérida, Venezuela: Universidad de los Andes. 2004. Asfaltenos ocurrencias y propiedades. p. 1-48.

7. Evdokimov IN, Losev a. P. On the Nature of UV/Vis Absorption Spectra of Asphaltenes. Pet Sci Technol. 2007;25(1-2):55-66.

8. Kharrat AM, Indo K, Mostowfi F. Asphaltene Content Measurement Using an Optical Spectroscopy Technique. Energy \& Fuels [Internet]. 2013;27(5):2452-7. Available from: http://dx.doi. org/10.1021/ef400050y

9. Östlund JA, Wattana P, Nydén M, Fogler HS. Characterization of fractionated asphaltenes by UV-vis and NMR self-diffusion spectroscopy. J Colloid Interface Sci. 2004;271(2):372-80.

10. Cardenas A, Bravo J, Blanco C, Briceño M, Espinoza C, Acevedo S, et al. Process of reducing viscosity of heavy crude oil by removal of asphaltene using a precipitating agent. United States; US2013/0264247 A1, 2013. p. 1-5.

11. Abdel K, Kentaro I, Farsshit M. Asphaltene content measurement using an opticalspectroscopy. Energy Fuels. 2013.

12. "Standard Test Method for Determination of Asphaltenes (Heptane Insolubles) in Crude Oil", ASTM D6560-00, an American National Standard, vol. 5. 2000. p. 1-6. 